\title{
Cancer Nanotechnology: Use of Smart Nanomaterials for Improved Outcome in Cancer Therapy
}

\author{
Devika Chithrani \\ Department of Physics and Astronomy, University of Victoria \\ Canada
}

In the battle against cancer, surgery, radiation and chemotherapy remain as the most widely used treatment options. Despite recent progress in conventional methods, there is still an immense need for new treatments that can eradicate cancerous cells while causing much less damage to the healthy tissue. Introduction of high atomic number materials such gold nanoparticles (GNPs) within the tumor could enhance the local radiation dose while minimizing the damage to surrounding tissue. In addition to this, NP-based technology has the capability to develop novel multiplex systems to combine more than one treatment modality for creating a more aggressive and effective approach in eradicating cancer. However, consideration of all three interfaces: in vivo delivery, tissue penetration, and successful delivery to individual tumor cells can play a bigger role in their future success. It is known that in vitro data cannot be extrapolated directly to in vivo or clinical settings. However, three dimensional tissue models can be used to create certain tumor microenvironment conditions for testing the potential of Nanotherapeutics before using them in vivo models. Such optimizations could lead to better results and would accelerate clinical use of Nanotherapeutics. I will discuss how we exploited three dimensional tissue models along with gold nanoparticle as a model nanoparticle system to improve the bio-nano interface for improved outcome in cancer therapy at this conference. 